\title{
Polysemy, a Scientific Issue in the Learning and Teaching of Foreign Languages
}

\author{
Arben Skendaj Ph.D \\ Faculty of Foreign Languages, University of Tirana \\ Email: arskendaj@hotmail.com
}

Doi:10.5901/jesr.2013.v3n2p265

\begin{abstract}
By this study it is aimed the solution of problems in the teaching of lexis in Italian and any other language. From a didactic and educational viewpoint it is aimed the students' acquaintance with these levels of research, the students' improved ability with these tasks and studies of similar nature so that they may be able to solve on their own problems in the field of culture and civilization. Polysemy in the general framework in Albania in the teaching and learning of foreign languages. The difficult problematic of polysemy in the learning of foreign languages and languages in general in intermediate, upper-intermediate and advanced level. An in-depth analysis of its explanation using scientific and research means. Polysemy is a lexical and semantic mechanism, which is a key to the enrichment of a foreign language dictionary, as well as to the Albanian one. In the framework of teaching of foreign languages, the lexis remains a very difficult aspect to solve, because there are involved other important teaching processes such as education, civilization which are not considered as primary goals. The scientific research will be done through a detailed research in the Italian language dictionaries and in literary texts. The methodology is focused on the analysis of the polysemantic structure of words from the viewpoint of etymology, grammar, semantics, meaning definition, examples and their phraseology. I will also illustrate it based on theoretical literature.
\end{abstract}

Key words: polysemy, expressions, idioms, semantics, meaning

\section{La polisemia come problema scientifico.}

Perché la polisemia si manifesta come un grande potenziale tecnico linguistico. Quando diciamo linguistico teniamo presente la lingua materna e le lingue straniere come lingua due, tre, quattro ecc. La polisemia sia nell'ambito della lingua madre, che delle lingue straniere è una chiave per entrare con dignità nella conoscenza dei problemi linguistici connessi alla comprensione e alla semanticità. La polisemia presenta un suo dupplice " carattere " linguistico, come moneta a due facce. Una è il lato numerico e l'altra è astratto, semantico. " La semantica viene definita tradizionalmente, a partire da Breal, come la scienza o la teoria dei significati " ( Guida alla semantica, pagina 7, George Mounin, Feltrinelli Economica 1983 Milano ). Il lessico di una lingua si presenta come la massa solida di una roccia, monolita. Esso non è assolutamente immune anche da modifiche e variazioni piccoli, lessico-semantici, il suo nocciolo ha una stabilità molto alta nel tempo e determinate sue parti difficilmente possono cambiare e qui ho presente il lessico di base dell'italiano nel caso nostro, e di ogni lingua in generale dal punto di vista lessicale sia quantitativo che qualitativo riferendosi alla semantica. Questo lessico non può cambiare facilmente, solo molto relativamente e riferito al tempo in fatto di cambiamenti si può parlare anche di secoli addirittura.

La polisemia come parte della semantica non può essere facile da trattare, " molti linguisti continuano a ripetere, che questa organizzazione dei significati benché vista attraverso il lessico resiste a ogni analisi esaustiva di tipo strutturale" ( "Guida alla semantica ", pagina 40, Geourge Mounin, Feltrinelli Economica 1983 Milano ).

Il lessico a prima vista si presenta con un numero di determinate parole e significati e sembra che questo suggerisca una situazione piuttosto lineare. In effetti la lingua gestisce l'aspetto semantico, quello dell'utilità pratica, della necessità di comunicare e da questo noi deduciamo che la lingua presenta l'aspetto semantico del lessico, cioè il numero dei significati supera di gran lunga quello delle parole. Il rapporto parole e significati in una lingua in genere rispecchia un rapporto, giustificato dalla polisemia, che approssimativamente dovrebbe 
aggirarsi su rapporto di 1 a 3, dove 1 sta per le parole e 3 per i significati, per esempio il vocabolario Zingarelli del 2013 vanta un numero di voci di 143.000 voci con 377.000 significati, dove vediamo che a 143.000 voci non corrispondono altrettanto significati. Questo succede in tutte lingue in francese, inglese, tedesco, spagnolo, russo ecc. I dizionari di queste lingue illustrano nelle copertine che oltre alla cifra delle voci c'è una cifra quasi tre volte maggiore di significati, e questa cifra giustamente è stata inserita solo da alcuni decenni, perché si considera come anche lo è, un criterio scientifico molto apprezzato per un'opera lessicografica qual'è il vocabolario di una lingua. Questa parte del lavoro lessicografico di un dizionario non è assolutamente in secondo piano, anzi è la parte più difficile, quella dei significati delle parole. Che dopo poter vedere che difficoltà e problematicità presenterebbe l'aspetto della definizione delle parole dal punto di vista semantico questo sarebbe proprio sapere i trucchi del mestiere, i vocabolari vanno valutati anche in base a facilità di comprensione derivante dalla definizione semantica che è diretto merito del lessicografo. Ma lasciamo stare questo argomento, che è un altro paio di maniche da trattare.

Tra la presentazione delle parole sotto l'aspetto fonetico, grammaticale e semantico sembra che ci sia un legame interiore, "naturale". Anche Mounin sostiene questo rapporto "La situazione non è assolutamente la stessa, che sta nelle parti formali della lingua, quelle che sono interessate fonetica, morfologia, e alla sintassi. In questi settori si è riusciti spesse volte e senza fatiche che si attivassero le strutture, legate alle funzioni della lingua e le hanno descritte"

( "Guida alla semantica", pagina 5, Geourge Mounin, Feltrinelli Economica 1983 Milano ).

Se non ci fosse la polisemia, questo meccanismo linguistico complicato, allora un vocabolario per esempio quello dell'italiano invece che di essere di 143.000 voci sarebbe di 377.000 voci nel caso che non ci fosse la polisemia a gestire la semantica dei significati, ma fosse la monosemia, cioè che a ogni parola ( combinazione fonematica ) appartenesse un solo significato. "Pur essendo stati fatti dei calcoli secondo i quali la nostra lingua ( l'italiano) possiede circa 300.000 e 500.000 parole di cui circa 100.000 sono parole di uso comune " ( Maurizio Dardano" Introduzione nell'italiano moderno, le strutture. Laterza, Bari 1993, pagina 296 )

In caso contrario la totalità dei significati di una lingua sarebbe gestita poi dalla monosemia e per ogni significato avremmo una parola sola e specifica, ma a quanto pare per la lingua è più pratico gestire la sua semantica delle parole con la polisemia, che con la monosemia. A vedere esternamente sembra che la polisemia avrebbe difficoltà a reggere tanti significati in una sola combinazione fonematica qual'è la parola, e che forse la monosemia avrebbe un compito più facile da svolgere. Invece questo compito si risolve molto bene dalla polisemia, essa gestisce definitivamente la semantica di una lingua e ha una sua tecnica specifica nel farlo e questo lo vedremo successivamente.

Il fenomeno della polisemia universale per molte lingue funziona come meccanismo linguistico in tutte lingue europee e allo stesso modo, cioè a determinate parole non appartengono determinati significati, ma molti di più, anche se penso che sia superfluo sostenere che non ci sono regole fisse per questo, cioè che nella lingua ci sono parole monosemiche, e il resto sono polisemiche con 2 o più significati senza che con questo volessi concludere erroneamente, che ci fossero delle regole prestabilite per il numero dei significati delle parole polisemiche. Come si gestisce una parola semanticamente non si gestisce un'altra.

Però perché questo si realizzi non è facile e ci sono dei meccanismi per realizzarlo, la polisemia attraverso le sue leggi interne semantiche e l'unità semantica nella struttura dei significati riesce a presentarsi come un meccanismo stabile, solido e funzionale, senza escludere però l'idea, che la semantica non va esclusa in maniera assoluta da un cabiamento molto relativo nel tempo; " ma i rapporti semantici, che esistono tra una parola e le parole vicino al sistema cambiano continuamente e attraverso il tempo" ( John Lyons "Manuale di semantica ", 1980, Laterza \& Figli, Bari, pagina 273 ).

Vista in questo prisma possiamo sostenere che la polisema sia nella lingua madre che nelle lingue straniere assume un'importanza di qualità nell'apprendimento e nell'insegnamento delle lingue straniere. Certamente quando diciamo importanza di qualità abbiamo presente l'apprendimento e l'insegnamento delle lingue straniere a livello elementare, medio e avanzato. E questo livello di conoscenza del lessico apre la porta a manipolare una lingua straniera, per gustarsi la sua letteratura, per sfruttare, capire, assimilare informazioni nei vari campi di scienze sociali quali storia, filosofia, sociologia, psicologia, giurisprudenza, antropologia, giornalismo, scienze politiche, archeologia ecc. Ecco cosa sostiene Mounin in merito a questo; " Come sistema la semantica è stata esclusa, teoricamente e praticamente dalla linguistica e abbandonata alla psicologia, 
antropologia culturale ( scuola americana), alla logica (Russel, Carnap ecc.) " Guida alla semantica ", pagina 24, Geourge Mounin, Feltrinelli Economica 1983 Milano ).

Gli aspetti dell'insegnamento della tecnica di arricchire il lessico presentano difficoltà abbastanza difficili da superare.

Ma la capacità nella gestione di un insegnante o di un professore sta nel sapere come distinguere, selezionare il valore, la frequenza di questi significati, spiegare agli studenti il modo, la tecnica concreta come possono individuare da soli il valore pratico della polisemia. Perché agli studenti una parola polisemica appare come standard e indistiguibile dalla importanza e dalla frequenza. Questo perché non tutti i significati sono uguali e non possono essre imparati in blocco e perché la memoria è quella che è ed ha i suoi limiti, specie se parliamo di una lingua straniera e quando si passa dal livello medio a quello avanzato di conoscenza della lingua straniera, sopprattutto bisogna riconoscere che non è facile affrontare questo impegno enorme di memorizzazione.

\section{L'importanza della ricerca.}

La mia modesta esperienza personale didattica e lessicale di 25 anni di insegnamento dell'italiano si basa sull'insegnamento della materia di tipologia testuale, lessicologia italiana (come corso teorico e pratico ), italiano parlato, l'italiano per finalità specifiche ( indirizzo master ). Ma va da sè che le varie materie hanno specificità diverse a essere insegnate e l'arricchimento del lessico, che è una delle strategie di base nell'insegnmento delle lingue straniere cambia in qualità e in quantità da materia a materia. Perché non ci facciamo illusioni certo che a livelli avanzati di insegnamento di una lingua straniera il fine primario diventa una tematica da imparare, un'informazione da prendere, però senza una buona conoscenza del lessico è impossibile progredire nello studio di questa lingua. Per esempio nella materia di tipologia testuale la polisemia viene insegnata anche come rubrica finalizzata e a parte, dedicata, ma indirettamente anche nelle altre rubriche; della sinonimia arricchita, differenziata, dell'antonimia, della conoscenza delle parole contestuali, delle domande interpretative e letterarie sul testo ecc. Si vuole riuscire ad arricchire il più possibile il lessico di questa lingua straniera da studiare, anzi è inevitabilmente utile. Mentre nella materia della lessicologia la polisemia viene spiegata sia sul piano teorico come su quello pratico, attraverso esercizi lessicali, che mirano intenzionalmente ad arricchire il vocabolario. $\mathrm{E}^{\prime}$ certo che in varie parole la polisemia presenta una diversa situazione polisemica, un diverso arricchimento semantico: alcune parole dispongono di 3,4,5,6,7,8 fino a decine di significati, senza contare l'aspetto semantico, ma solo quello formale della quantità dei significati, specifico per ogni parola, perchè tecnicamente la difficoltà non è la stessa per ogni parola come abbiamo già detto.

Possiamo affermare che la polisemia sia nella lingua madre che in quelle straniere presenta una situazione sovraccarica di significati, sfumature, usi, illustrazioni, fraseologie, espressioni sintagmatiche e idiomatiche che ci aiuta a districarsi nell'intricato mondo dei significati per lo più di una lingua straniera e manipolarla sia a livello passivo di conoscenza che attivo di parlarla." La polisemia è un fenomeno di grande rilievo nella strutturazione del lessico almeno per due ragioni; 1 . Realizza un risparmio molto grande delle parole: 2 aiuta a ricordare con maggiore facilità le parole. Vediamo adesso alcune frasi dove viene usato il verbo passare ( con diversi significati in diversi contesti ):
a. Il ladro passa per la finestra "entra"
b. Mario passa a casa di Pietro "va"
c. La pasta passa di cottura "supera il limite della cottura"
d. L'alunno passa un esame "lo supera"
e. Il tempo passa "va avanti"
f. Il raffreddore passa "va via"
g. Quell'incosciente passa in curva "sorpassa"
h. La spada lo passa da parta a parte "lo attraversa" ( La Nuova Grammatica della Lingua Italiana, Maurizio Dardano e Pietro Trifone, Zanichelli, BolognaEditore 2001, pagina 29.)

Le parole polisemiche hanno pure loro la loro etimologia, e qui mi riferisco all'etimologia semantica, che può essere conservata, trasformata, cambiata nel tempo, in sfumature, usi ecc., ma un nocciolo semantico può anche essere stato conservato. Così la parola visita, occhio, orecchio, andare ecc. si suppone, che nel tempo abbiano 
avuto lo stesso significato essenziale, che hanno oggi. Queste parole, se volessimo immaginare una situazione figurata, potremmo crederla come un treno sul quale salgono e scendono persone, porta e manda via merci, viaggia in varie direzioni, ma esso i binari e qui mi riferisco ai principali significati e sfumature semantiche di esse sono le stesse, non cambiano la loro essenza semantica facilmente, i loro semi nucleari non vanno via. Per esempio parole così possiamo citare; casa, correre, tempo, bello, buono, cattivo, sole, luna ecc.

Le parole polisemiche se facessimo un bilancio e verifica apparirebbero talmente semplici a prima vista altrettanto difficili e complicate risulterebbero poi. Esse sono le prime parole ad essere imparate sia nella lingua madre che in quelle straniere, perché sono indispensabili e molto frequenti. Esse in ogni lingua appartengono al lessico più frequente e più usato. Le parole polisemiche di una lingua possiamo presupporre, che sono la parte base del lessico di una lingua e possiamo considerarle anche come i "fossili" della lingua in maniera figurata cioè della sua lontana origine. Anche la parola fossile qui io l'ho usata linguisticamente, figuratamente, potenzialmente all'infuori della sua struttura semantica consolidata, perché mi dà un'idea simbolica, che molte parole non me la darebbero ed è anche un uso sporadico, non codificato semanticamente, linguisticamente. "

Spesso le classificazioni di tipo sinonimico e antonimico rispecchiano tutta la struttura delle conoscenze di una civiltà, sia nei vocabolari etimologici, nei quali i rapporti tra i significati vengono costruiti da successive derivazioni dai significati delle parole in diversi periodi storici" ( "Guida alla semantica ", pagina 43, Geourge Mounin, Feltrinelli Economica 1983 Milano ).

La polisemia è una delle vie principali per arricchire il lessico di una lingua, perché come sappiamo alle lingue va più a genio di sovraccaricare piùttosto una parola già polisemica, di un secondo, terzo, quarto, quinto, sesto, settimo significato ecc. che a creare una parola nuova quando necessita di usare un neologismo. "La nozione della polisemia, che è centrale nell'esame degli spostamenti dei significati..." ( Gaetano Berruto, "La semantica ", pagina 62, Zanichelli, Bologna, 1979).

La creazione, l'invenzione di una nuova parola espone la lingua, la società, l'uomo a situazioni spesso non serie, ridicole e insicure. Perciò la polisemia è spesso una via lessico-semantica molto tecnica, perfetta per risolvere i problemi, che emergono nei suoi aspetti neologici. "A quanto pare infatti il progresso tecnico è un fattore della polisemia" ( Maurizio Dardano "Introduzione all'italiano moderno, le strutture", Laterza, Bari, 1993, pagina 305 )

Spesso tenendo presente, che dal punto di vista semantico la polisemia è molto ricca allora anche quando ci saranno parole derivate da esse queste nuove unità lessicali molto probabilmente trasporteranno molti dei suoi aspetti polisemantici delle parole da cui derivano. Da questo possiamo concludere che in questi casi la polisemia è possibile che si trasferisca nella nuova parola, ma questo non è detto mai in maniera assoluta, perchè la nuova parola è stata creata sì da una parola già esistente, ma avrà anche una sua vita autonoma, per cui forse qualche significato non corrisponderebbe, qualche nuovo può essere stato usato; comunque in genere si avverte una tendenza di mantenere la polisemia della parola da cui è stata derivata. Durante i processi di derivazione e di formazione di nuove parole con l'aiuto dei prefissi e dei suffissi queste parole sono esposte a sfumature, la loro semantica può essere destinata ad allontanarsi dalla fonte semantica iniziale. Vediamo un gruppo simile solo come esempio; il verbo condurre ( in italiano significa portare, guidare, mandare, fare ), dove il participio passato condotto che significa anche "tubi di acqua", condotta che significa "comportamento" , e così di seguito avremo una lista di derivati della parola condurre che sono; condottiero, conducibile, conduzione, conduttura, conduttore, conduttivo, conduttività, conduttanza, ricondurre, riconducibile ecc. E che hanno significati relativamente diversi.

Visto sul piano teorico e pratico questo studio sulla polisemia sulla lingua madre e quelle straniere potrebbe servire anche come spunto per fare studi, analisi, lauree, dottorati e ricerche varie. Sul piano pratico poi possiamo pure affermare, che la polisemia e la sua conoscenza sembra che non porti all'arricchimento del lessico di una lingua perché quando l'uomo impara un nuovo significato, una nuova sfumatura di una parola già saputa o una sua nuova fraseologia sembra che esso non impara una nuova parola !! Però riconsiderando l'idea di prima, che la polisemia è un meccanismo linguistico, che arricchisce il lessico evitando di creare una parola nuova, cioè di avere parole monosemiche, ma sceglie la via del sovraccaricare di un nuovo significato una parola già esistente nella lingua effettivamente possiamo concludere, che questo è come se si imparasse una nuova 
parola, e risultasse indubbiamente come un effettivo arricchimento del lessico di una lingua caso nostro dell'italiano.

\section{La metodologia della ricerca.}

Le difficoltà dell'arricchimento del lessico e concretamente quello dell'arrichimento della polisemia possono essere risolte anche seguendo queste vie; cioè leggendo continuamente e sotto controllo didattico, e con controllo intendo dare agli studenti rubriche di lavoro, di testi letterari di scrittori italiani e di materiali dei campi sociali in generale; questa potrebbe essere una via comoda per arricchire e memorizzare il lessico polisemico e il lessico di una lingua straniera e sopratutto quando si è in una fase di conoscenza da un livello elementare a quello medio e avanzato, da un primo impatto di questo lessico a un suo nuovo spontaneo incontro in contesti naturali, letterari rafforza questo lessico, lo stabilizza dalla posizione dello studente, che si imbatte nella conoscenza ed arricchimento del lessico di una lingua straniera.

Nella materia della tipologia testuale I'arricchimento semantico, polisemantico viene realizzata in molti piani. Al secondo corso dell'indirizzo di italianistica nella Facoltà delle Lingue straniere, all'Università di Tirana dove io insegno, la concezione della materia viene basata su un livello medio, post-medio e avanzato della lingua straniera.

La rubrica della sinonimia entra direttamente anche nella filtrazione e chiarificazione dei problemi polisemici, perché in questa rubrica vengono prese parole scelte di un testo letterario, preferibilmente racconti di scrittori italiani, delle categorie grammaticali del verbo, sostantivo e dell'aggettivo. Vengono scelte parole di una certa frequenza non molto alta, perché queste parole appartengono al lessico sconosciuto e che hanno anche una polisemia non eccessiva, di circa 2,3,4,5 significati. Vengono ricercati sinonimi contestuali per la parola scelta e a volte è risultato che ci siano stati anche 20 sinonimi contestuali; condizione assoluta è stato solo il contesto come riferimento per essere sinonimi. In seguito bisogna fare le differenze semantiche tra la parola del testo con i suoi sinonimi fuori dal contesto e indirettamente si troverà anche la ricchezza polisemica. Questa differenza sinonimica viene fatta attraverso analisi delle loro strutture semantiche, attraverso illustrazioni delle varie sfumature semantiche, letterarie, contestuali, la frequenza dell'uso.

Poi nella rubrica finalizzata della polisemia, sempre in questa materia di tipologia testuale, viene chiesto di lavorare con parole di un alto tasso di frequenza di uso, si mira di imparare la loro struttura principale semantica con i suoi contesti e fraseologie principali.

La polisemia semanticamente gestisce una situazione abbastanza complessa. Essa si presenta a prima vista con una struttura per quanto grande e sufficientemente consolidata dal punto di vista semantico; ci sono suoi aspetti semantici potenziali, che non possono essere figurati neanche nei dizionari. Questa sua parte si erige al di sopra dello standard lessico-semantico. Questo uso potenziale è esteso a tutte le lingue ed a un livello avanzato. Questo "uso" direi non è "ufficialmente" linguistico. Questi usi lessico-semantici potenziali appartengono alle sfere letterarie, che sono al confine della metafora, della simbolica, metonimia, sineddoche ecc. "Tradizionalmente vengono distinte tre categorie principali del trasferimento del significato; metonimia, sineddoche e metafora ..." ( Gaetano Berruto, "La semantica ", pagina 116, Zanichelli, Bologna, 1979).

Questi usi sono potenziali; una ragione perché non vengono registrati nei vocabolari significa che questi riferimenti semantici possiamo utilizzarli in caso di "lusso" per fare un effetto linguistico, letterario, figurato, emozionale, momentaneo, colloquiale, umoristico, satirico, che lo standard linguistico normale non lo adempie, non lo risolve. Parole come queste possiamo citare; visita, reminiscenza, casa, resto, occhio, simbiosi, appassionare, apparenza, lottare, dignità ecc. Vale a dire che questi usi sono usi appartenenti a una intelligenza intellettuale media e post-media e avanzata. Così la parola visitare normalmente ha i significati incontrare qualcuno per intrettenersi in chiacchere o passare il tempo per amicizia, gentilezza oppure andare in un posto per ragioni di lavoro, svago, studio, preghiera e simili per esempio "Visiterò Parigi settimana futura" . L'espressione carta da visita, oltre alla semantica standard, come fraseologia che significa un pezzo di carta, dove sono segnati i dati di una persona, nome, cognome, indirizzo, mestiere, i contatti ecc. Possiamo suggerire che in maniera figurata, potenziale possiamo usarla anche per indicare la parlata di una persona, per il suo modo di vestire, per il suo comportamento al primo impatto verso un'altra persona o più persone per esempio " Lui doveva apprezzare che il suo comportamento era come una specie di carta da visita perché non lo conoscevamo" 
La parola casa ha i suoi significati consolidati; luogo, abitazione per persone, animali, origine, posto per ragioni di svago, studio, preghiera ecc., ma possiamo usarla anche con ironia, scherzo, sarcasmo ecc, p.s. " per lui il lavoro è diventato la sua casa" e sottintendiamo ironicamente, che una persona ha perso il concetto del referimento della casa come tale, e che sta pochissimo a casa e il maggior tempo lo passa fuori essa, Quando vogliamo dire di una persona che ci fa una bella sorpresa, e gentilmente e delicatamente noi vogliamo dare maggiore importanza diciamo "ma guarda che bella visita abbiamo avuto!" mentre potevamo dire, "ma guarda chi è venuto!", oppure " Come mai da queste parti oggi!" ecc.

Questi usi simbolici e figurati possono riguardare sia sostantivi, aggettivi che verbi.

Dal punto di vista lessicale ed etimologico l'italiano è in un certo senso " un latino moderno" o l'ereditaria più "fedele" al latino soprattutto per il fatto geografico, cioè che il latino è nato ed è stato usato nel territorio dell'attuale Italia oltre alla costatazione linguistica che l'italiano rispecchia realmente più fedeltà grammaticale e semantica al latino. Tra le altre lingue neolatine e l'italiano noi troviamo molto corrispondenze lessicosemantiche e altrettante coorrispondenze lessico-semantiche noi troviamo per le stesse parole polisemiche latine e neolatine nell'italiano e lingue come l'albanese e l'inglese, lingue non appartenenti alla famiglia delle lingue neolatine il cui lessico è di circa $65 \%$ latino e neolatino. Ecco come si esprime Dardano in merito " L'italiano di oggi partecipa in condizioni, che sono comuni per le altre lingue europee" (Maurizio Dardano " Introduzione nell'italiano moderno, le strutture" Laterza, Bari 1993, pagina 324)

Volendo studiare ulteriormente i meccanismi interni della polisemia, oltre gli aspetti trattati io direi che le espressioni fraseologiche ed idiomatiche, facenti parte delle parole polisemiche, sono un ulteriore arricchimento del lessio semantico della polisemia. Esse sono delle unità molto particolari da molti punti di vista e presentano un grande interesse linguistico e queste unità le racchiude e le gestisce sempre la polisemia. Tentiamo di poter analizzare, spiegare i loro lati storici, e dell'uso.

Costatiamo che la maggior parte delle espressioni sintagmatiche, fraseologiche ed idiomatiche "sono rifugiate" in seno alle parole polisemiche. Questo indica però che la polisemia ha anche questo aspetto lessicosemantico, essa lo gestisce nella sua struttura. Le fraseologie sono una "storia" o "ministoria" determinata nell'ambito della lingua. Le fraseologie possono essere composte da due o più unità lessicali. Nei vocabolari esse vengono registrate nelle parole polisemiche, che fanno parte delle fraseologie e che sono al centro dell'idea, dell'attenzione semantica. Queste fraseologie in un certo senso possono essere considerate anche come "significati", che si aggiungono alla polisemia delle parole. Le ragioni e le circostanze del perché nascono queste unità freseologiche, viste nell'ambito della polisemia, sarebbe di grande interesse e curiosità trattarle. Così per esempio in italiano l'espressione idiomatica "andare a Canossa" significa "fare atto di sottomissione umiliante, ritrattandosi e riconoscendo la supremazia dell'avversario. Questo però deriva da una data storia verificata nel medioevo dove a Canossa che era centro in provincia di Reggio Emilia, famoso per il suo castello, nel quale, all'epoca della lotta per le investiture, l'imperatore Enrico IV si recò (gennaio 1077) per ottenere, umiliandosi in veste di penitente, il perdono di papa Gregorio VII, ospite della contessa Matilde, proprietaria del castello. Di qui la frase fig. "andare a Canossa." (Vocabolario Treccani online). Come si vede a uno sfondo storico reale l'espressione concreta per il suo tempo ha preso in seguito una funzione linguistica semantica quella succitata. In italiano di casi di locuzioni idiomatiche provenienti dal passato e dalla storia ne se trovano a bizzeffe, anche se non è sempre possibile rintracciare l'etimologia come il caso di "andare a Canossa". Queste espressioni sono un'alternativa all'uso delle parole, ma non sono equivalenti, certamente servono nella lingua ad offrire una semantica che le parole non la trasmettono.

Potremmo fare una distinzione tra le espressioni sintagmatiche, fraseologiche e idiomatiche qual'era la precedente quella di andare a Canossa, che sono cariche di un peso figurato talmente alto da non poter dedurre sempre facilmente il suo significato. Le espressioni sintagmatiche sono facilmente deducibili come significato, sono frutto piuttosto di una somma matematica delle unità lessicali usate. Per esempio l'espressione "a parole", che significa riferito alle parole e non ai fatti per esempio "Lui è bravo a parole, ma i fatti lo smentiscono", a occhio a misura, di colpo, in seguito ecc. Queste espressioni sono abbastanza frequenti, quasi insostituibili nella lingua e suggerirei di imparare in italiano se si vuole pretendere di sapere bene questa lingua. Le espressioni idiomatiche sono una via di mezzo tra i due gruppi che si assomigliano per certi aspetti, ma per altri si differenziano, queste lucuzioni sono meno frequenti delle espressioni sintagmatiche e più usate delle idiomatiche, completano la conoscenza di una lingua straniera; come quelle idiomatiche coloriscono molto I'italiano caso nostro, però non sono certo la prima barriera da abbattere in una lingua straniera qual'è l'italiano. 
Questi tre gruppi di fraseologie non possono essere esauriti qui come argomento da trattare, perché richiederebbero una sede, occasione e spazio apposito da poter essere consumato, però accennarlo mi è sembrato doveroso, visto che parlavamo di polisemia.

\section{I risultati della ricerca.}

Volendo fare uno scanner alla polisemia diremo che è una via molto semplificatrice ed orientativa per disctricarsi nell'intricato terreno del lessico della lingua madre e di una lingua straniera. Questa analisi e suggerimento possono essere visti anche come un modo per orientarsi nella consultazione e gestione del lessico del vocabolario di una lingua, perché il vocabolario è l'archivio più preciso della lingua e in questo ambito possiamo citare " Uno dei componenti principali della linguistica è sempre stata la lessicografia, cioè lo studio dei significati delle parole e la compilazione dei vocabolari" ( "La semantica, le strutture della lingua" Gennaro Chierchia, Mulino. Bologna 1977, pagina 194). Questi aspetti risultano molto utili all'apprendimento e nell'insegnamento delle lingue straniere, mentre uno studente, un insegnante o professore resta molto indubbio o incerto di fronte al vocabolario, ma questo, se si permette è un aspetto chiave nella conoscenza di una lingua straniera. Perché per quanto uno possa pretendere di conoscere bene una lingua straniera poi in fin dei conti, non può gustarsi la sua letteratura e neanche la lettaratura specifica se non conosce bene il lessico, altrimenti sacrificherà parti del messaggio non comprenderà a fondo il materiale da consultare.

Io credo in fine che dopo queste analisi e valutazioni della polisemia e del suo ambito linguistico nelle lingue madri e quelle straniere non posso pretendere di essere stato esaustivo per la sua problematicità così complicata. Essa ha molti lati, aspetti, angolazioni dalle quali si può analizzare. Io credo che questa analisi aveva il dupplice scopo di orientare gli interessati nell'oceano infinito del lessico, come di educare, di abilitare e familiarizzare chi è interessato con le ricerche scientifiche.

Ecco cosa dice John Lyons in merito alla struttura del lessico di una lingua: " $E^{\prime}$ vero che il lessico di una lingua è stato strutturato in maniera gerarchica in termini di iponimia" (Lyons, J. 1980. Manuale di Semantica. Pagina 323. Bari:Laterza \& Figli.). La mia analisi e studio sono stati orientati intenzionalmente su aspetti piuttosto teorici che pratici, che sono anche più facili da analizzare, non che abbia trascurato esempi pratici, ma quelli teorici sono anche i problemi più spinosi, sono le prime difficoltà da superare

\section{Bibliografia}

Mounin, G. (1983). Guida alla semantica. Milano: Feltrinelli Economica.

Lyons, J. (1980). Manuale di semantica. Bari:Laterza \& Figli.

Dardano, M. (1993). Introduzione nell'italiano moderno. Le strutture. Bari:Laterza.

Chierchia, G. (1997). La semantica, le strutture della lingua. Bologna: Mulino.

Berruto, G. (1979). La semantica. Bologna: Zanichelli.

Dardano, M. \& Trifone, P. (2001). La Nuova Grammatica della lingua italiana, Bologna: Zanichelli Editore.

Lo Zingarelli, (1994).Vocabolario della lingua italiana. Bologna: Zanichelli editore, S.p.A. Via Irnerio 34, 40126.

De Voto-Oli (2004). Dizionario della Lingua Italiana. Firenze: Felice Le Monnier, Edmund Le Monnier S.p.A.

Fjalor i Gjuhes Shqipe, (2006). Akademia e Shkencave e Shqiperise. Tirane: Instituti i Gjuhesise dhe i Letersise.

The New Oxford Dictionary of English, (2001). Oxford: Edited by Judy Pearsall, Oxford University Press.

Enciclopedia Treccani, Vocabolario Treccani, online. 2013.. 
